Published in "Advances in Science and Technology vol. 45. pp. 2448-2458 (2006)"

\title{
Iterative method in the characterization of piezoceramics of industrial interest
}

\author{
L. Pardo ${ }^{1, a}$, M. Algueró ${ }^{1, b}$ and K. Brebø| ${ }^{2, c}$ \\ ${ }^{1}$ Instituto de Ciencia de Materiales de Madrid (ICMM-CSIC). Cantoblanco. \\ 28049 - Madrid (Spain) \\ ${ }^{2}$ Limiel ApS. Langebæk. (Denmark) \\ alpardo@icmm.csic.es, ${ }^{b}$ malguero@icmm.csic.es, kkreboel@post3.tele.dk
}

Keywords: piezoceramics, resonance, impedance measurements, iterative methods, finite element modelling, matrix characterization, dielectric properties, elastic properties

Abstract. Although characterization of piezoceramics from resonance is a customary technique, the works dealing with the determination of the ten elastic, dielectric and piezoelectric coefficients that are needed in the full matrix characterization of such $6 \mathrm{~mm}$ symmetry materials are rather scarce. Even more, if the complex characterization is foreseen, thus accounting with the three types of losses, few are the methods avaliable to obtain the material linear coefficients. This work deals with such complex characterization by the use of Alemany et al. automatic iterative method. This method has been applied to the four modes of resonance that are sufficient for the purpose: (1) the length extensional mode of long rods, length poled, (2) the thickness extensional mode and (3) the radial mode of a thin disk, thickness poled, and (4) the thickness shear mode of a thin plate. Recent work of the authors has shown the limits in characterizing materials that arise from the use of the Standard shear geometry and, therefore, and alternative geometry is used here. This work presents the matrix characterization of some piezoceramics and the Finite Element Analysis (FEA) simulation based on such characterization, of the samples used as a reliability criteria of the results obtained by comparison of the experimental and simulated values at resonance of the electrical parameters.

\section{Introduction}

The modeling and design of new piezoelectric devices by, among other numerical methods, the finite element analysis, relies on the accuracy of the dielectric, piezoelectric and elastic coefficients of the active material used, commonly an anisotropic ferroelectric polycrystal, or piezoelectric ceramic. The polarization in the randomly oriented grains of an as-processed ferroelectric ceramic can be oriented in the direction of an external electric field, in the so-called "poling" process (Fig.1), giving as a result an induced anisotropy of the properties and a piezoelectric effect [1]. 
The accurate description of piezoceramics involves the evaluation of the dielectric, piezoelectric and mechanical losses, accounting for the out of phase material response to the input signal, which is not always accomplished despite of their important role in the material performance. Losses in piezoceramics have inconvenient consequences for positioning actuator applications, since they lead to hysteresis in the field-induced strain, and for resonance applications, such ultrasonic motors, since they are the cause of heat generation. On the other hand, they can be an advantage for force sensors and acoustic transducers, since they widen the frequency band for receiving signals. The description of the material parameters by complex values $\left(\mathrm{P}^{*}=\mathrm{P}^{\prime}-\mathrm{i} \mathrm{P}^{\prime \prime}\right)$ is a convenient way to separately account for the dielectric, piezoelectric and mechanical losses (tan $\delta=$ $\left.P^{\prime \prime} / P^{\prime}\right)$. The origin of the losses in ferroelectric ceramics has been analyzed in numerous works [2-5].

Poled ferroelectric ceramics in their normal operating range show substantially linear relations between the stress $\left(T_{i j}\right)$ and the strain $\left(S_{i j}\right)$, which are tensor magnitudes, on the one hand and between the electric field $\left(\mathrm{E}_{\mathrm{i}}\right)$ and the dielectric displacement $\left(D_{i}\right)$, which are vector magnitudes, on the other. Besides, the piezoelectric coefficients provide relations between mechanical and electrical magnitudes [1]. These relationships can be written making use of different sets of coefficients in reduced matrix form. One of these sets, frequently used, is:

$$
\begin{aligned}
& S=s^{E} T+d_{t} E \\
& D=d T+\varepsilon^{\top} E
\end{aligned}
$$

where $s$ is the matrix of elastic compliance, $d$ is the matrix of piezoelectric charge coefficients and $d_{t}$ its transposed (changing rows by columns) matrix, and $\varepsilon^{\top}$ the dielectric permittivity matrix. Superindex E means constant (zero) field and superindex T means constant (zero) stress.

\section{The resonance method}

Characterization methods for bulk piezoceramics from impedance measurements at the electromechanical resonances, providing their full dielectric, piezoelectric and elastic complex coefficients matrices in the linear range, have been used since early times of the development of these materials in the 60 's $[1,7]$, and become a widespread practice. In the frequency range of an electromechanical 
resonance, the resonator of a given geometry has electrical impedance, $Z$, which depends on the frequency, the dimensions of the sample and its density, and a given set of dielectric, piezoelectric and elastic coefficients. For this reason, the values of the coefficients can be obtained from impedance measurements as a function of the frequency (Fig. 1) on a suitable shaped sample, provided that the analytical solution of the wave equation for the mode of motion of that sample is known. The first standard procedures of measurements on piezoceramics date from 1961 [8] and were issued by the North American "Institute of Radio Engineers (IRE)". The aim of this Standard was to adapt previous definitions, relations and measurement methods, developed for piezoelectric crystals in general, to the characteristics of those new materials.

The electromechanical coupling factor, $k$, is defined as the square root of the energy transformed by the resonator divided by the total energy input, and expresses the ability of the resonator to transform electric energy into mechanical energy, and vice versa. The resonator can be described by an LCR (inductance, capacitance, resistance) resonant circuit. As it is well known, the complex impedance of the resonator is $Z=R+i X$, where $X$ is the reactance. The mechanical quality factor, $Q_{m}$, is defined as the expression of the internal mechanical damping of the resonator and by the ratio between the circuit reactance and resistance. The higher the mechanical quality factor, the lower the mechanical losses of the resonator.

The complex admittance of the resonator is defined as the reciprocal of the impedance and as $\mathrm{Y}=\mathrm{G}+\mathrm{iB}$, where $\mathrm{G}$ is the conductance and $\mathrm{B}$ the susceptance. Standard defines $f_{n}$ and $f_{m}$ as the frequencies of maximum and minimum impedance, respectively, in the neighborhood of the resonance, and also defines the procedures (the so-called transmission line, or transmission circuit, method) to measure the resonator impedance and determine these frequencies. Coupling coefficients and quality factors of the resonator are calculated from the difference between these two frequencies. Standard also defines other pairs of frequencies: $f s$ and $f p$, the series and parallel frequencies, as the frequencies for maximum $G$ and maximum $R$, respectively, and $f_{r}$ and $f_{a}$, the resonance and antiresonance frequencies, as the frequencies of $B=0$ and $X=0$, respectively. The values of these frequencies are such that (Figure 2 ):

$$
\left(f_{n}-f_{m}\right)>\left(f_{p}-f_{s}\right)>\left(f_{a}-f_{r}\right)
$$


The main piezoceramic characteristics considered in the development of 1961 Standard were the high symmetry $(6 \mathrm{~mm})$, leading to simpler relations than for most piezoelectric crystals, high electromechanical coupling constants, low mechanical quality factors and noticeable dielectric loss factor $\left(\tan \delta=\varepsilon^{\prime \prime} / \varepsilon^{\prime}\right)$. If such conditions are fulfilled, the following approximations are valid:

$$
\left(\mathrm{f}_{\mathrm{n}}-\mathrm{f}_{\mathrm{m}}\right) \approx\left(\mathrm{f}_{\mathrm{p}}-\mathrm{f}_{\mathrm{s}}\right) \approx\left(\mathrm{f}_{\mathrm{a}}-\mathrm{f}_{\mathrm{r}}\right)
$$

This first Standard was reviewed and updated several times, being the most recent one issued by the North American institutions "American National Standards Institute (ANSI)" and "The Institute of Electrical and Electronic Engineers (IEEE)" in 1987 [9]. Although with a clear explanation of their limitations, the 1987 IEEE Standard still is based in the approximations given above in (3).

There is at present a general knowledge of these limitations, however, 1987 IEEE Standard is still widely in use. Their validity holds for many of the most widely used commercial piezoceramics based on lead titanate zirconate (PZT) compositions, which are low- $Q_{m}$ and high-coupling coefficients piezoelectric materials. But, there is a general agreement that their use in many new piezoelectric materials, like porous ceramics, piezoelectric polymers or piezoelectric composites leads, when applicable, to important errors. Furthermore, IEEE Standard does not account for the complex nature of the material coefficients, keeping the dielectric loss factor $(\tan \delta)$ and the mechanical quality factor $\left(Q_{m}\right)$ as the only parameters accounting for the losses in materials. The dielectric permittivity is "traditionally" obtained from the sample capacitance measured at $1 \mathrm{kHz}$. This is not a good practice. It is also well known that there is a change in permittivity, elastic compliance and piezoelectric response with frequency, which is more pronounced in ferroelectric materials. Variations between such permittivity value at $1 \mathrm{kHz}$ and that included in the analytical solution of the wave equation corresponding to a resonance taking place at a given frequency (from $100 \mathrm{kHz}$ to $10 \mathrm{MHz}$ ) are, thus, assumed. Recommendation for the use of such low frequency, also included in 1987 IEEE Standard for $\varepsilon^{\top}{ }_{\mathrm{ij}}$, is made on the basis of avoiding interfering with the capacitance measurement of the lowest resonance's of plates, usually taking place at $\mathrm{f} \sim 100 \mathrm{kHz}$. However, 1987 IEEE Standard contributions to the characterization of piezoelectric ceramics are, of course, remarkable. The Standard states the resonator geometries and the relationships among their dimensions for the 
validity of the equations used in the IEEE Standard and all the relationships among the coefficients needed to get all the independent coefficients for piezoceramics. Four modes of resonance (Table 1) of three sample shapes are sufficient for this purpose $[10]$.

\section{Iterative methods in the complex characterization of piezoceramics}

One of the first attempts to treat losses in piezoceramics by considering complex coefficients is due to Holland and dates from 1967 [11]. Already Holland and EerNisse [12] proposed a gain-bandwidth method for length extensional mode of thickness poled bars, which was valid for moderate losses and low electromechanical coupling coefficient materials. Since then, many papers on the topic have been published, some of which will be referred to in the following. Smits [13] published the first iterative procedure for the accurate determination of complex materials coefficients. The major drawback of the method is that it requires a skilled operator, because the judicious choice of the 3 frequencies for measurement of $Z$ is needed, in order to avoid the determination of constants with large errors. From such Z values a system of non-linear equations is established and solved in the corresponding coefficients by an iterative procedure. The first automatization and the extension to complex parameters of the procedure by Meitzler et al. [14], adopted in the 1987 IEEE Standard [9], for a piezoelectric resonator in the radial mode of a thin disk, was published by Sherrit et al. [15]. This is the most mathematically complex resonance mode. These authors also introduced a refinement of the 1987 IEEE Standard method by a polynomial fit, easier to implement in terms of automatization and more accurate, instead of the linear interpolation, used in the IEEE Standard, for the data of the Poisson ratio, $\sigma^{P}$, as a function of the ratio of the first overtone to the fundamental resonance frequencies, $f_{s}^{(2)} / f^{(1)}{ }_{s}$, in the determination of $\sigma^{P}$. These authors also proposed a non-iterative evaluation method, and applied it to thickness extensional plate resonators [16]. This method implicitly predetermines that the phase of the electromechanical coupling factor is only dependent on the mechanical loss. It is only applicable for materials with low dielectric and piezoelectric losses. Sherrit et al. accomplished as well the first complete material characterization in a complex form by the resonance method of a commercial ceramic [17]. They used four geometries and 
five resonance modes: the ones shown in Table I, and the length extensional mode of a thickness poled bar. For that purpose they used their own, non-iterative, method for the radial mode of a thickness poled thin disk resonator and the Smits method for the rest of the modes.

Alemany et al. $[18,19]$ developed an automatic iterative method, based on Smits one, that overcomes its main drawback by the automatization of the choice of the frequencies for the measurement of $Z$ (or $Y$ depending on the resonance mode). This method is the only iterative method that to date that was systematically applied to all resonance modes needed for the full matrix characterization of piezoceramics (Table 1). Alemany et al. method has been also applied to the determination of the parameters from overtone resonances in the radial and thickness modes of thin disks [20], thus accounting for a discrete determination of the dispersion of the complex material parameters.

In 1997 Kwok et al. [21] published a comparative study, applied to thickness extensional plate resonators. The methods compared were: a non-linear regression method proposed by the authors (the Gauss-Newton fitting method), a commercial software (the Piezoelectric Resonance Analysis Program (PRAP), based on Smits [13] and Sherrit et al. [16] works), the 1987 IEEE Standard method, the Smits method and the Sherrit et al. methods. Together with other moderate loss materials (copolymers, lead metaniobate ceramics and composites), they characterized polyvinylidene fluoride (PVDF), a high-loss piezoelectric polymer. They found the already mentioned limitations of such methods, i.e. that 1987 IEEE Standard, Smits and Sherrit et al. methods, although requiring skilled operators, are valid methods for materials with moderate losses but fail in the characterization of high-loss materials. As for any other fitting method, the proposed non-linear regression method has the drawback of being sensitive to the choice of the data segment used. Each calculated material parameter represents an average within the frequency range where data points are used for the calculation. The narrower the fitting range the closer are the calculated average values to the actual values of the parameters.

The interest that the topic of the characterization of piezoelectric materials with losses drags nowadays is shown by the very recent publications on new fitting 
methods, although just applied to length extensional mode of thickness poled bars [22, 23].

\section{Iterative automatic method developed by C. Alemany et al. at ICMM-CSIC}

In this method the coefficients are calculated as the solution - obtained by an automatized iterative numerical method - of the set of non-linear equations that results when experimental impedance, (or admittance) data are introduced into the analytical solution of the wave equation for the mode of motion of the sample. Such impedance or admittance data are taken at four given frequencies in the neighborhood of the resonance, being the determination of such frequencies also automatic. This analytical solution exists for all the four resonance modes (Table I) needed for the determination of the complete set of parameters and given in Figure 12. The impedance for the thickness resonance of a disk poled and excited along its thickness is given by the equation:

$$
Z=R+i X=-i \frac{t}{2 \pi f S \varepsilon_{33}^{s}}+i \frac{h_{33}^{2}}{2 \pi^{2} S f^{2} c_{33}^{D} \sqrt{\frac{\rho}{c_{33}^{D}}}} \tan \left(\pi f t \sqrt{\frac{\rho}{c_{33}^{D}}}\right)
$$

where $S=\pi(D / 2)^{2}$ (Figure 12) is the electroded surface area and $\rho$ is the ceramic density.

The admittance for the radial resonance of a disk poled and excited along its thickness is given by:

$$
Y=G+i B=i \frac{2 \pi^{2} f D^{2}}{4 t}\left(\varepsilon_{33}^{T}+2 d_{31}^{2} \frac{\frac{c_{11}^{p}}{2-\mathfrak{I}_{1}\left[\pi f D \sqrt{\frac{\rho}{c_{11}^{p}}}\right]}-\frac{1}{1+\sigma^{p}}}{(\pi)}\right)
$$

where $t$ is the thickness of the disk (Figure 12 ), $\sigma^{P}$ is the Poisson ratio, $\sigma^{P}=-s_{12}{ }^{E} / s_{11}{ }^{E}$, $\mathrm{C}_{11}{ }^{\mathrm{P}}$ is a relationship among constants, $\mathrm{C}_{11}{ }^{\mathrm{P}}=\mathrm{s}^{\mathrm{E}}{ }_{11}\left[\left(\mathrm{~s}_{11}^{\mathrm{E}}\right)^{2}-\left(\mathrm{s}_{12}^{\mathrm{E}}\right)^{2}\right]^{-1}$, and $\mathfrak{J}$ is the Onoe function [15] defined as:

$$
\mathfrak{S}_{1}[z]=\frac{z J_{0}(z)}{J_{1}(z)}
$$


being $J_{0}$ and $J_{1}$ the Bessel functions of the first kind and zero and first orders, respectively.

The impedance for the shear resonance of a plate poled across and excited along its thickness is:

$$
Z=R+i X=-i \frac{t}{2 \pi f S \varepsilon_{11}^{s}}+i \frac{h_{15}^{2}}{2 \pi^{2} S f^{2} c_{55}^{D} \sqrt{\frac{\rho}{c_{55}^{D}}}} \tan \left(\pi f t \sqrt{\frac{\rho}{c_{55}^{D}}}\right)
$$

where $S=L w$ (Figure 12) is the electroded surface area.

The impedance for the length resonance of a bar poled and excited along its length is given by:

$$
Z=R+i X=-i \frac{L}{2 \pi f S}\left(\frac{1}{\varepsilon_{33}^{T}}+\frac{g_{33}^{2}}{s_{33}^{D}}\right)+i \frac{g_{33}^{2}}{2 \pi^{2} S f^{2} s_{33}^{D} \sqrt{\rho s_{33}^{D}}} \tan \left(\pi f L \sqrt{\rho s_{33}^{D}}\right)
$$

where $S=\pi(D / 2)^{2}$ (Figure 12) is the electroded surface area.

Only the dimensions and density of the sample, together with the value of the complex admittance or impedance at such four frequencies around the resonance are required to get the dielectric, elastic and piezoelectric complex coefficients that rules in each mode of resonance. Just in the radial mode of thin disks it is additionally needed to know the value of $\mathrm{f}^{(2)}$ of the first overtone. In practice, an experimental data file of absolute values of admittance, $\left|Y_{i}\right|$, and its phase angle, $\theta_{i}$, at each frequency, $f_{i}$, is obtained in a frequency interval around the resonance. From these values, the corresponding values of conductance, $G_{i}=|Y|_{i} \cos \theta i$, and resistance $R_{i}=\cos \theta_{i}|Y|_{i}^{-1}$ are obtained. Two of the four frequencies involved in the calculation, $f_{s}$ and $f_{p}$, are determined by location of the maximum values of $R_{i}$ and $G_{i}$ in the measured interval, and the values of complex impedance at such frequencies introduced in the system of non-linear equations to be solved, in the so-called central iteration of the method. The determination of the other two frequencies involved in the calculation of the central iteration, $f_{1}$ and $f_{2}$, constitutes a second iterative process, called peripheral iteration of the method, which finish when the convergence of $f_{2}$ fulfills the criteria: $\mid f_{2}\left(\right.$ final) $-f_{2}$ (initial) $\mid<0.05 \%$. The details concerning the two iterations of Alemany et al. method are given in references 18 and 19. As a quality criteria of the results obtained 
by the method, both the $\mathrm{G}$ and the $\mathrm{R}$ profiles are reconstructed by insertion of the obtained complex coefficients in the analytical solution of the wave equation of the given resonance mode and by variation of the frequency. Typically, when the experimental results are free of spurious resonances, corresponding to a single resonator, the regression factor between the experimental and reconstructed values is higher than 0.99 .

In the first publications on the method, the complex characterization of a number of commercial piezoceramics (Ferroperm Piezoceramics A/S), with high (PZ27, lead zirconate titanate) and low (PZ34, modified lead titanate, and PZ45, bismuth niobate) coupling coefficients and with high (PZ27) and low (PZ35, lead metaniobate) mechanical quality factors, was accomplished. Although still fully valid, some of the calculation details given in the first works have benefited from the long experience of application of the method to a wide number of ceramic materials [24-27] and has been refined to be easier to use in the research and industrial characterization and quality control laboratories.

\section{Matrix Characterization of Piezoceramics using Non-Standard Shear Samples}

Widely used for device modeling, the Finite Element Analysis (FEA) has been scarcely used in materials science. The strong potential of this tool in the study, for example, of the modes of motion of the material resonators remains unexplored, among other reasons, by the lack of complete set of data, including losses, for the piezoceramics of industrial interest. The modeling has been restricted to consider these as materials without losses [28] and isotropic media [29].

Difficulties in getting a coherent set of material parameters from resonance data on different sample shapes arise on the dispersive character of the piezoceramics and the dependence of their properties on the polarization level [1]. Whereas the radial and longitudinal extensional resonances of thin disks and long rods, respectively, take place typically in the range of hundreds of $\mathrm{kHz}$, the thickness extensional and shear resonances of thin disks and shear plates can be currently found in the range of few $\mathrm{MHz}$. Besides, to achieve the saturation level of polarization in a long bar or in an in-plane poled shear plate is frequently more difficult than in a thin disk. The resonance method for these reasons has suffered criticisms and alternative methods 
to obtain the full ensemble of coefficients were developed using ultrasonic spectroscopy [30], a method not responsive to all losses.

Despite of all this, careful poling and accurate determination of complex properties by the systematic use of Alemany et al. method to the three sample shapes and four resonance modes of Table 1, have recently lead to a reliable full complex characterization of a soft, Navy II-type, lead zirconate titanate commercial piezoceramic (PZ27 Ferroperm Piezoceramics A/S)[10] and a high sensitivity Mn-doped Morphotropic Phase Boundary 0.655 Pb( $\left.\mathrm{Mg}_{1 / 3} \mathrm{Nb}_{2 / 3}\right) \mathrm{O}_{3}-0.345 \mathrm{PbTiO}_{3}$ ceramic [27].

The availability of the full set of coefficients, including all losses, has allowed the modeling by 3-D FEA of PZ27 piezoceramic items [31,32] of the three geometries used for the matrix characterization. This work have the double purpose of assess the validity of the material constants, by comparing modeled and experimental impedance data, and to study the modes of resonance of the three Standard samples used in the characterization (Fig. 2). Secondary resonance peaks (Fig. 3) appearing in resonance spectra of the in-plane poled Standard shear plate (Fig. 2(b)) are commonly observed, independently of the composition and dimensions of the shear sample, while keeping the Standard dimensional ratios $L, w \geq 10 t$, and often related to material inhomogeneities, such as those due to inhomogeneous poling caused by edge effects. The results of the FEA analysis, corresponding to an elastically, dielectrically and piezoelectrically homogeneous item of PZ27, shows [31] that in fact this explanation is not correct and that secondary modes can also be modeled at frequencies in good agreement with the measured ones (Fig. 3-left). Secondary peaks take place due to the occurrence of non purely-shear modes in such a Standard sample geometry and dimensional ratios. FEA modeling reveals that, for this particular shear sample, at $\mathrm{f}=1790 \mathrm{kHz}$ the mode of motion resembles a composition of a thickness shear and an asymmetric Lamb wave modes of motion with perpendicular propagation directions, whereas at $f=1440 \mathrm{kHz}$, the mode of motion is even more complex [31]. The shear coefficient d15 reported for PZ27 seems to be underestimated, $d_{15}=(396-26 i) p C . N^{-1}$, by the iterative calculation from impedance measurement at resonance of the Standard sample [10] when compared to the actual value quoted by the manufacturer $\left(d_{15}=500\right.$ pC. $\mathrm{N}^{-1}$ ), which is obtained from direct measurements on accelerometers working with shear elements [33]. FEA results also show that, at the mode of motion for the main 
resonance, the shear displacement in the center of the sample is much higher than the one at the edges of the Standard shear item, showing that the sample is dynamically clamped (Fig. 3-right). Measurements of normal displacement to the surface of the Standard shear resonator by laser interferometry have confirmed that the modes of motion of the Standard shear sample are a composition of plane waves and the inhomogeneous shear displacement [34]. These results also reveal the dynamical clamping as the source of the underestimation of the material parameters when using Standard shear items. The poling problem may be an additional difficulty when characterizing piezoceramics using Standard shear samples, because they result in resonance spectra with double peaks that cannot be treated as a single resonance, using formula (7). This was observed for PZT based ceramics with close porosity [35] and some PMT-PT ceramics (Fig. 4(up)) [27]. The use of a non-Standard shear sample (Fig. 2(f)), to make use of the second thickness shear resonance suggested by Berlincourt [7], is at present under test. Unwanted multiple resonances are avoided using this sample (Fig. 4(down)) and higher piezoelectric shear coefficients are obtained. Additionally, this non-standard shear sample allows characterization from identically poled samples at the thickness and at the radial resonances of thin disks (Fig. 2(d)) and shear resonance of shear plate (Fig. 2(e)).

\section{Summary}

A critical literature review of methods for characterization of piezoceramics of commercial interest is presented, from the 1961 Standards to the most recent fitting and iterative methods, taking into account material losses. Alemany et al. automatic iterative method is to date the only one systematically applied to all sample shapes and resonances needed for the determination of the full ensemble of characteristic complex material parameters. Matrix material characterization, including losses, allows the study of material resonators by Finite Element Analysis. The modes of motion of the satellite and principal resonances of the Standard shear item were analyzed. The dynamical clamping of the Standard shear main resonance was found to explain the underestimation of the piezoelectric material shear coefficients. A proposal of an alternative, non-Standard, shear sample, making use of the second thickness shear mode of resonance, is made aiming to enhance the coherence and reliability of the 
matrix characterization using the Alemany et al. method from three samples and four modes of resonance.

\section{Acknowledgements}

This work was carried out under projects MAT 2001-4819-E and the Ramon y Cajal Program of the Spanish $\mathrm{CICYT}$, and has benefited from the synergy provided by the POlar ELEtroCERamics, POLECER, (G5RTCT2001-05024) Thematic Network of the EC. Authors wish to thank Ms. W.W.Wolny and Dr. E. Ringaard from Ferroperm Piezoceramics A/S (Kvistgård, Denmark) for the ceramic samples used. Dr. C. Alemany and Mr. A. García (holder of a POLECER-CAM co-funded FINNOVA 2004 grant at ICMMCSIC) are acknowledged for some of the measurements carried out for this work. Authors are also indebted to Dr. C. Alemany (ICMM-CSIC, at the time of writing this paper already retired) for the generous implementation of the automatic iterative method to the second thickness shear geometry and thoughtful discussions of the work.

\section{References}

[1]“Piezoelectric Ceramics”. B. Jaffe, W.R. Cook and H. Jaffe. Academic Press. London, 1971.

[2] K.H. Haerdtl. "Electrical and mechanical losses in ferroelectric ceramics". Ceram Int. 8, 121(1982).

[3] K. Uchino and S. Hirose." Loss mechanisms in piezoelectrics: How to measure different losses separately" IEEE UFFC 48, 307(2001).

[4] G. Robert, D. Damjanovic and N. Setter. "Piezoelectric hysteresis analysis and loss separation". J. Appl. Phys. 90(9), 4668(2001).

[5] A.V. Mezheritsky. "Elastic, Dielectric and Piezoelectric Losses in Piezoceramics: How It Works All Together" IEEE Ultrasonics, Ferroelectrics and Frequency Control, 51(6), 695-707 (2004).

[6] "Physical Properties of Crystals. Their representation by tensors and matrices". J.F. Nye. Oxford at the Clarendon Press. London, 1957.

[7] "Piezoelectric and Piezomagnetic Materials and Their Function in Transducers". D.A. Berlincourt, D.R. Curran and H. Jaffe in "Physical Acoustics" vol.1 Part A. Ed W.P. Mason, Academic Press, 1964.

[8] "IRE standard on piezoelectric crystals: Measurements of piezoelectric ceramics, 1961", Proc. IRE, 49(7), 1161-1169 (1961).

[9]“IEEE Standard on piezoelectricity". ANSI/IEEE Std. 176-1987.

[10] M. Algueró, C. Alemany, L. Pardo and A. M. Gonzalez. "Method for Obtaining the Full Set of Linear Electric, Mechanical and Electromechanical coefficients and All Related Losses of a Piezoelectric Ceramic". J. Am. Ceram. Soc. 87(2), 209 (2004) [11] R. Holland. "Representation of dielectric, elastic and piezoelectric losses by complex coefficients". IEEE Trans.Sonics Ultrason. SU-14(1),18 (1967).

[12] R. Holland and E. EerNisse. "Accurate measurement of coefficients in a ferroelectric ceramic". IEEE Trans.Sonics Ultrason. SU-16(4),173 (1969).

[13] J.G. Smits. "Iterative method for accurate determination of the real and imaginary parts of the materials coefficients of piezoelectric ceramics". IEEE Trans. Sonics Ultrason. SU-23(6),393-402(1976). 
[14] A.H. Meitzler, H.M. O'Bryan, Jr., and H.F. Tiersten. "Definitions and measurements of radial mode coupling factors in piezoelectric ceramic materials with large variations in Poisson's ratio" IEEE Trans.Sonics Ultrason. SU-20(3), 233 (1973).

[15] S. Sherrit, N. Gauthier, H. D. Wiederick and B.K. Mukherjee. "Accurate evaluation of the real and imaginary material constants for a piezoelectric resonator in the radial mode". Ferroelectrics 119, 17 (1991).

[16] S. Sherrit, H. D. Wiederick and B.K. Mukherjee. "Non-iterative Evaluation of the Real and Imaginary Material Constants of Piezoelectric Resonators". Ferroelectrics 134, 111 (1992).

[17] S. Sherrit, H.D. Wiederick and B.K. Mukherjee "A complete characterization of the piezoelectric, dielectric and elastic properties of Motorola PZT3203HD including losses and dispersion". Medical Imaging 1997: Ultrasonic Transducer Engineering, SPIE Proceedings, 3037, 158 (1997).

[18] C. Alemany, L. Pardo, B. Jiménez, F. Carmona, J. Mendiola and A.M. González. "Automatic iterative evaluation of complex material constants in piezoelectric ceramics". J. Phys. D: Appl. Phys. 27, 148 (1994).

[19] C. Alemany, A.M. González, L. Pardo, B. Jiménez, F. Carmona and J. Mendiola. "Automatic determination of complex constants of piezoelectric lossy materials in the radial mode". J. Phys. D: Appl.Phys. 28(5), 945 (1995).

[20] A.M. Gonzalez and C. Alemany." Determination of the frequency dependence of characteristic constants in lossy piezoelectric materials". J. Phys. D: Appl.Phys. 29, 2476 (1996).

[21] K.W. Kwok, H.L.W. Chan and C.L.Choy. "Evaluation of the Material Parameters of Piezoelectric Materials by various methods". IEEE Trans. on Ultrasonics, Ferroelectrics and Frequency Control 44(4), 733 (1997).

[22] T. Tsurumi, Y.B. Kil, K. Nagatoh, H. Kakemoto and Shatoshi Wada. "Intrinsic Elastic, Dielectric, and Piezoelectric Losses in Lead Zirconate Titanate Ceramics Determined by an Immittance-Fitting Method" J.Am.Ceram.Soc. 85(8), 1993 (2002).

[23] X. Hong Du, Q. M. Wang and K. Uchino. "Accurate Determination of Complex Materials Coefficients of Piezoelectric Resonators". IEEE Trans. on Ultrasonics, Ferroelectrics and Frequency Control 50(3), 312 (2003).

[24] J. Ricote, C. Alemany and L. Pardo. "Microstructural effects on dielectric and piezoelectric behaviour of calcium modified lead titanate ceramics". J. Mater. Res. 10(12), 3194 (1995).

[25] L. Pardo, P.Duran-Martín, J.P. Mercurio, L. Nibou and B. Jiménez."Temperature behaviour of structural, dielectric and piezoelectric properties of sol-gel processed ceramics of the system $\mathrm{LiNbO}_{3}-\mathrm{NaNbO}_{3}$ ". Journal of Phys. and Chem. Solids 58(9), 1335 (1997).

[26] A. Moure, C. Alemany and L. Pardo. "Temperature dependence of piezoelectric, electromechanical and elastic parameters of Aurivillius-type structure piezoceramics with optimized texture and microstructure". IEEE Trans. Ultrasonic Ferroelectrics and Frequency Control, 52(4), 570-577 (2005).

[27] M. Algueró, C. Alemany, L. Pardo and M.P. Thi. "Piezoelectric Resonances, Linear Coefficients and Losses of Morphotropic Phase Boundary $\mathrm{Pb}\left(\mathrm{Mg}_{1 / 3} \mathrm{Nb}_{2 / 3}\right) \mathrm{O}_{3}-\mathrm{PbTiO}_{3}$ Ceramics". Journal of the American Ceramic Society 88(10), 2780-2787 (2005).

[28] A. Barzegar, D. Damjanovic and N. Setter. "The effect of boundary conditions and sample aspect ratio on apparent $d_{33}$ piezoelectric coefficients determined by direct 
quasistatic method“ IEEE Trans. on Ultrasonics, Ferroelectrics and Frequency Control 51(3), 262-270 (2004).

[29] R. Steinhausen, T. Hauke, H. Beige, S. Seifert, U. Lange, D. Sporn, S. Gebhardt and A. Schönecker. "Characterization and modelling of ferroelectric thin films and 1-3 composites". Bol. Soc. Esp. Ceram. Vidrio 41(1), 158-165 (2002).

[30] H. Wang and W. Cao. "Determination of full set material constants from phase velocities". J. Applied Physics 92(8), 4578- 4583(2002).

[31] L. Pardo, M. Algueró and K. Brebøl. "Resonance modes in Standard piezoceramic shear geometry: a discussion based on Finite Element Analysis". Journal de Physique IV France 128, 207-211 (2005).

[32] L. Pardo, M. Algueró and K. Brebøl. "Resonance modes in Standard characterization of piezoceramics: a discussion based on Finite Element Analysis". Ferroelectrics, in press (2006).

[33] http://www.ferroperm-piezo.com

[34] L. Pardo, F. Montero de Espinosa and K. Brebøl. "Study by laser interferometry of the resonance modes of the shear plate used in the Standards characterization of piezoceramics" (submitted).

[35] L. Pardo, A. García, K. Brebøl, D. Piazza and C. Galassi. "Matrix Characterization, Including Losses, of Porous PZT based ceramics with Morphotropic Phase Boundary Composition: Key Issues"(submitted). 
Table 1. Resonance modes, sample shapes, and corresponding directly obtained coefficients for each mode, needed for the determination of the full characteristic matrices of a piezoelectric ceramic.

\begin{tabular}{|c|c|c|}
\hline Sample geometry & Resonance mode & $\begin{array}{l}\text { Directly obtained } \\
\text { coefficients }\end{array}$ \\
\hline $\begin{array}{l}\text { - thin disks, thickness poled } \\
\text { (Diameter>20xthickness) } \\
\text { - shear plates } \\
\text { (Length, width> 10xthickness) } \\
\text { - long bars, length poled } \\
\text { (Length > 10xDiameter) }\end{array}$ & $\begin{array}{l}\text { - thickness extensional mode of thin plates } \\
\text { - radial mode of thin disks } \\
\text { - thickness shear mode of thin plates } \\
\text { - length extensional resonance of long bars }\end{array}$ & $\begin{array}{l}\text { - } \mathrm{h}_{33}, \varepsilon^{\mathrm{S}}{ }_{33}, \mathrm{c}^{\mathrm{D}}{ }_{33} \\
\text { - } \mathrm{d}_{31}, \varepsilon^{\top}{ }_{33}, \mathrm{~s}^{\mathrm{E}_{11}, \mathrm{~s}_{12}} \\
\text { - } \mathrm{h}_{15}, \varepsilon^{\mathrm{S}}{ }_{11}, \mathrm{c}^{\mathrm{D}}{ }_{55} \\
\text { - } \mathrm{g}_{33}, \varepsilon^{\top}{ }_{33}, \mathrm{~s}^{\mathrm{D}}{ }_{33}\end{array}$ \\
\hline
\end{tabular}




\section{Figue captions}

Figure 1. Left: Schematic view of grains of a ferroelectric ceramic showing the polarization direction before (up) and after (down) the poling process. The 3 direction is arbitrarilly chosen as the one of the applied electric field. Right: Impedance (modulus, $|\mathrm{Z}|$, real, R, and imaginary, $X$, parts) dependence on frequency in the vicinity of an electromechanical resonance.

Figure 2. Above: The three sample shapes $(a, b, c)$ used in the Standards for matrix characterization. Below: Process to obtain, from a thickness poled disk (d) or plate (e), a nonstandard shear sample (f).

Figure 3. (Left) Experimental and FEA generated resistance, $R$, and conductance, $G$, for the fundamental shear thickness resonance of the plate of PZ27. (Right) Grey level shows shear (X) displacement of the Standard shear element with in-plane polarization at $1572 \mathrm{kHz}$.

Figure 4. Experimental $R$ and $G$ curves (symbols), and reconstructed profiles (lines) after Alemany et al. calculations, at the resonance of (a) an Standard shear plate and (b) nonStandard shear plate of $\mathrm{Mn}$-doped $0.655 \mathrm{~Pb}\left(\mathrm{Mg}_{1 / 3} \mathrm{Nb}_{2 / 3}\right) \mathrm{O}_{3}-0.345 \mathrm{PbTiO}_{3}$ ceramic. 

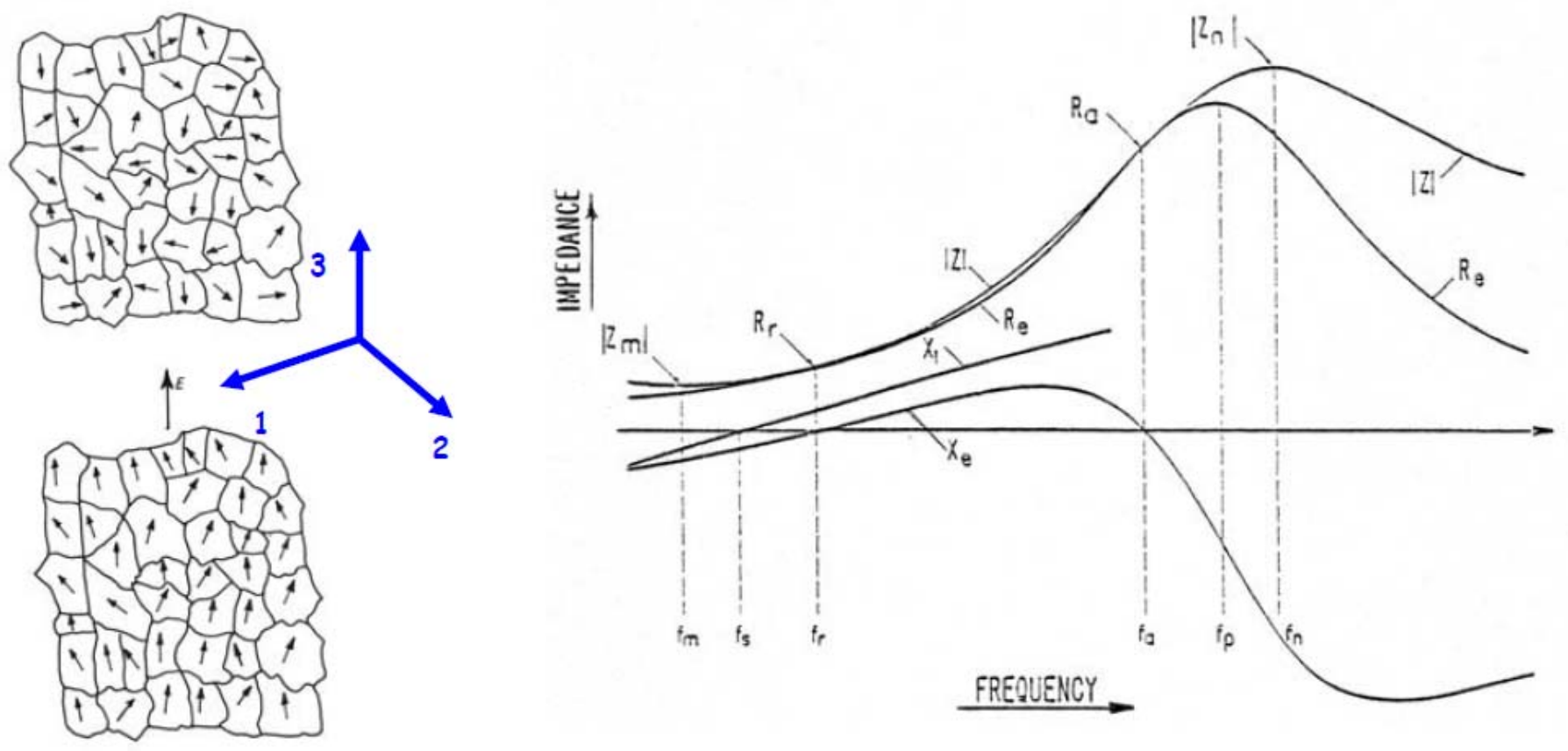

Fig. 1/4 in "Iterative method in the characterization..." by L. Pardo et al. 

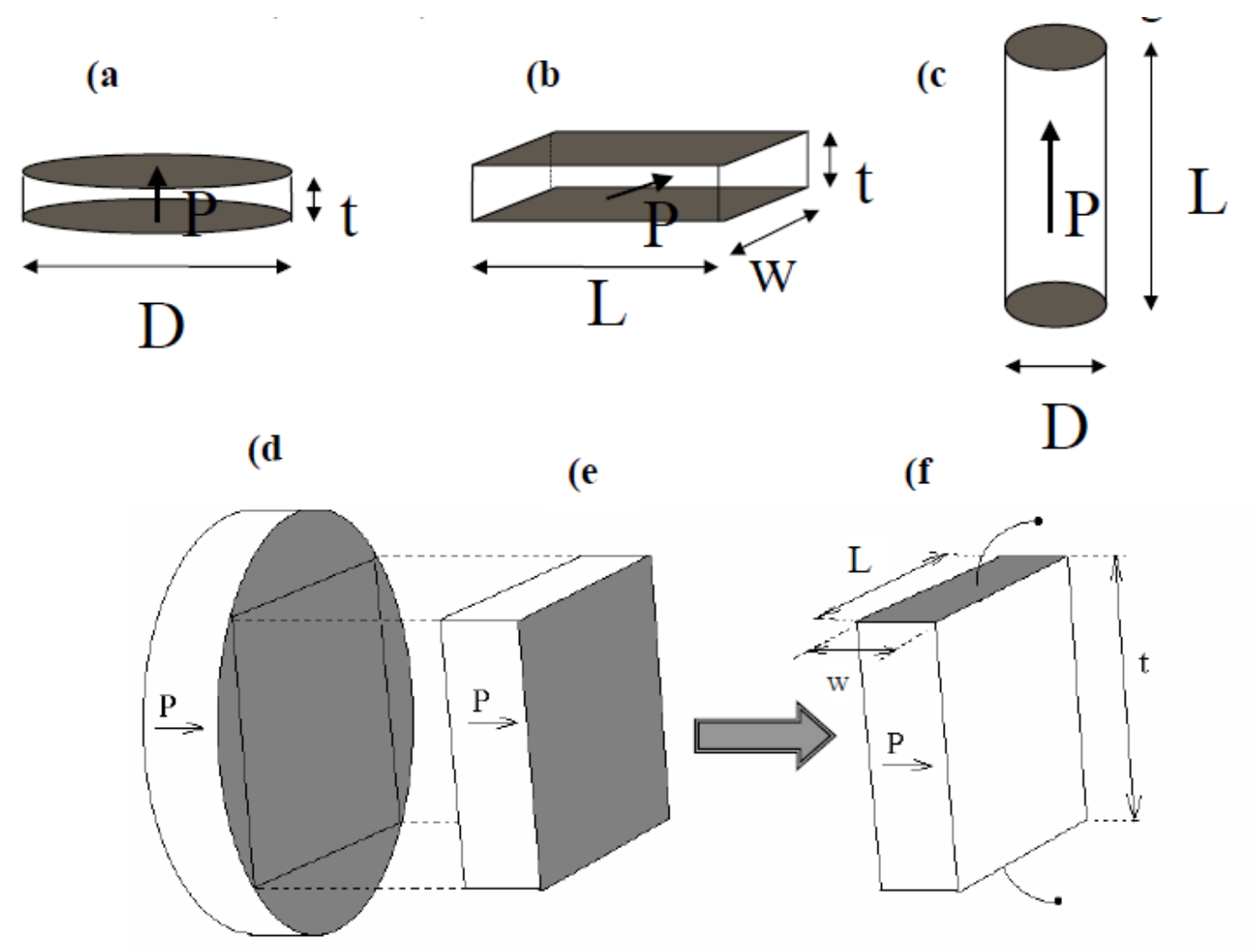

Fig. 2/4 in "Iterative method in the characterization..." by L. Pardo et al. 

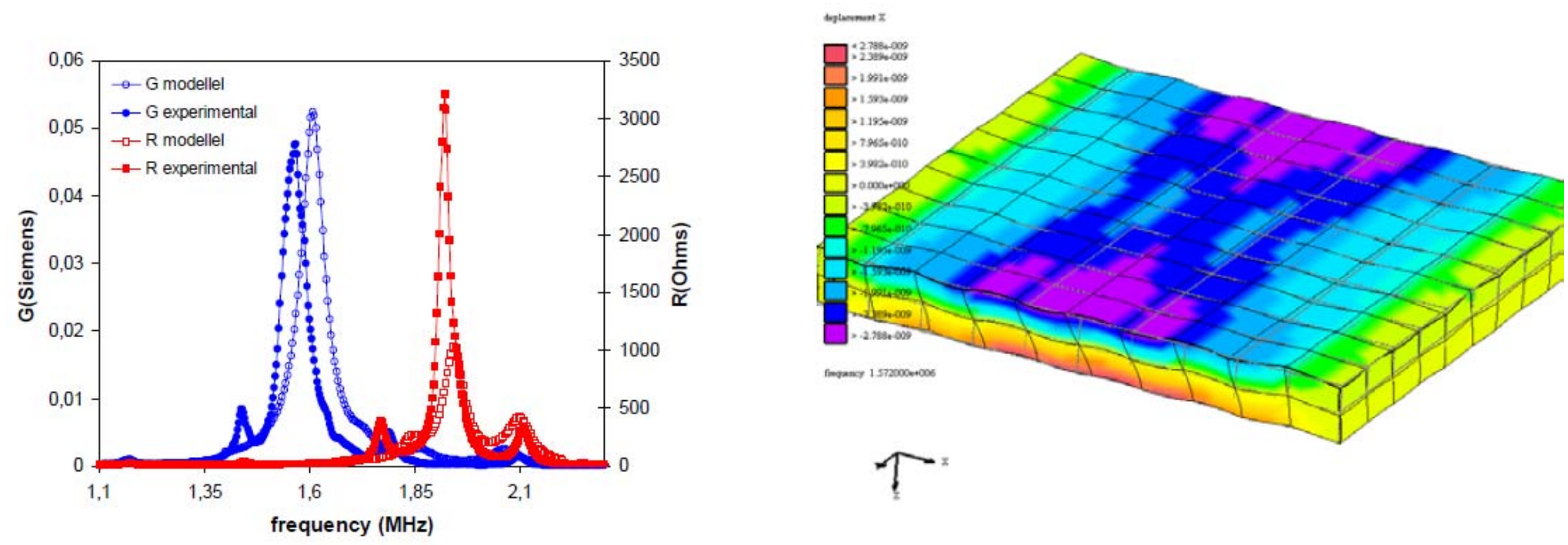

Fig. 3/4 in "Iterative method in the characterization..." by L. Pardo et al. 


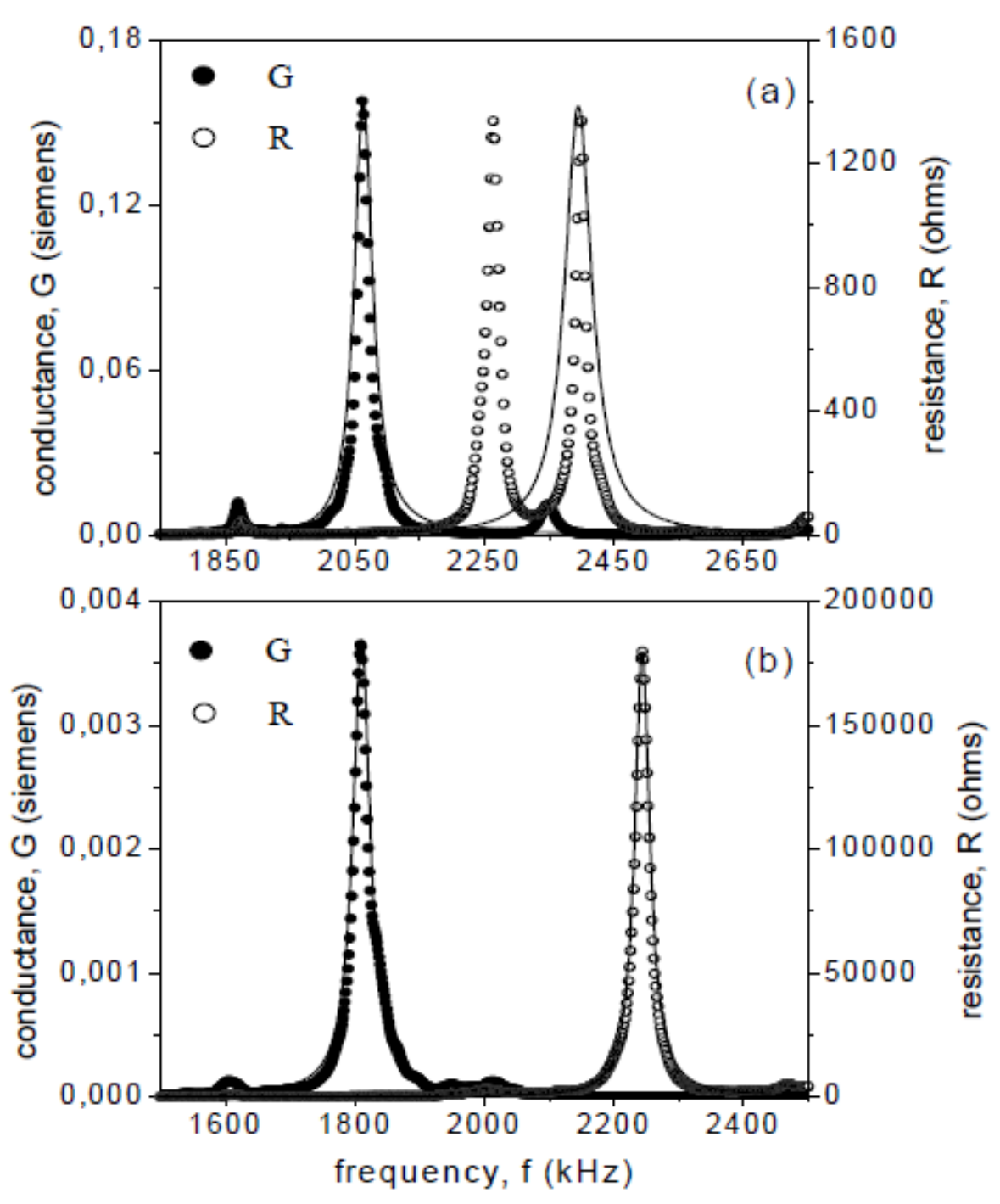

Fig. 4/4 in "Iterative method in the characterization..." by L. Pardo et al. 